\title{
Ezrin expression and its phosphorylation in gastric carcinoma with lymphoid stroma and Epstein-Barr virus infection
}

\author{
TARO TOBO $^{1}$, MINAKO HIRAHASHI ${ }^{1}$, TAKASHI YAO ${ }^{2}$, SHINICHI AISHIMA $^{1}$ and YOSHINAO ODA $^{1}$ \\ ${ }^{1}$ Department of Anatomic Pathology, Graduate School of Medical Sciences, Kyushu University, Fukuoka; \\ ${ }^{2}$ Department of Human Pathology, Juntendo University School of Medicine, Tokyo, Japan
}

Received July 13, 2012; Accepted December 5, 2012

DOI: $10.3892 / \mathrm{mco} .2012 .56$

\begin{abstract}
Gastric carcinoma with lymphoid stroma (GCLS) is a unique variant of gastric carcinoma that represents prominent lymphocytic infiltration and is correlated with Epstein-Barr virus (EBV) infection. Ezrin expression and activation are crucial in tumor metastasis and induce cell migration of EBV-related nasopharyngeal carcinomas. Using immunohistochemical methods, the expression of total and phosphorylated ezrin (p-ezrin), Thr567, was examined in 104 GCLS cases, including 78 EBV-positive and 26 EBV-negative cases, as well as 29 non-GCLS cases. Positive ezrin expression was detected to be at markedly higher levels in GCLS compared to non-GCLS $(\mathrm{P}<0.0001)$. Furthermore, ezrin expression was detected to be at higher levels in EBV-positive compared to EBV-negative GCLS ( $\mathrm{P}=0.0294)$. High expression of p-ezrin in GCLS was associated with positive lymph node metastasis $(\mathrm{P}=0.0187)$. In summary, these results demonstrated that ezrin overexpression is correlated with the histologic characteristics of GCLS and EBV infection. Phosphorylation of ezrin may, therefore, contribute to lymph node metastasis in GCLS.
\end{abstract}

\section{Introduction}

Gastric carcinoma with lymphoid stroma (GCLS) is rare due to the unique histological features of gastric carcinoma with a favorable prognosis (1). GCLS demonstrates a well-defined tumor border and contains several lymphocytes, plasma cells and lymphoid follicles (1). Lymphoepithelioma-like carcinoma demonstrates prominent lymphoplasmacytic infiltration and is commonly associated with Epstein-Barr virus (EBV) infection (2), which is well-known to be present in GCLS cancer cells (3-6).

Correspondence to: Dr Shinichi Aishima, Department of Anatomic Pathology, Graduate School of Medical Sciences, Kyushu University, 3-1-1 Maidashi, Higashi-ku, Fukuoka 812-8582, Japan

E-mail: saish@surgpath.med.kyushu-u.ac.jp

Key words: ezrin, gastric carcinoma, Epstein-Barr virus, lymphoid stroma
Ezrin is a member of the ezrin/radixin/moesin (ERM) family, and is characterized by small-sized molecules linking the plasma membrane and the actin cytoskeleton (7). Ezrin expression and phosphorylation are also crucial in the regulation of tumor metastasis (8-10). Ezrin expression is reduced in diffuse-type gastric carcinoma (11), while ezrin overexpression is known to be correlated with the progression and poor prognosis of gastric carcinomas $(12,13)$. Recently, ezrin phosphorylation was found to be associated with EBV latent membrane protein 1 (LMP1) and to induce tumor migration in nasopharyngeal carcinomas (14).

Ezrin expression has been identified in ordinary gastric carcinomas, whereas ezrin expression in GCLS and its correlation with EBV infection have not been previously studied. Therefore, the present study aimed to investigate ezrin expression and its phosphorylated form in GCLS and non-GCLS.

\section{Materials and methods}

Tissue samples. GCLS or without lymphoid stroma(non-GCLS) samples were surgically resected and diagnosed at the Department of Anatomic Pathology of the Kyushu University (Fukuoka, Japan) between 1970 and 2008. The study protocol used was in compliance with the Ethical Guidelines of the 1975 Declaration of Helsinki. For strict privacy protection reasons, information identifying the samples was removed prior to analysis. The GCLS group included 74 males and 26 females with a median age of 62.0 years (range, 37-90), whereas the non-GCLS group included 16 males and 13 females with a median age of 58.4 years (range, 39-82). GCLS was defined as a well-circumscribed and expansive growth pattern adenocarcinoma (Fig. 1A) with a dense and diffuse lymphoid infiltration (Fig. 1B), and a lace-like or small-nested proliferative pattern of carcinoma cells with various degrees of cellular pleomorphism (Fig. 1C), according to previously published studies $(1,3,4)$. Non-GCLS for the control cases was defined as medullary growth-type adenocarcinoma lacking frequent lymphoid infiltration or a glandular component. Subsequently, GCLS $(n=104)$ were collected from 100 patients and non-GCLS $(n=29)$ from 29 patients, based on the histopathological definition provided above.

In situ hybridization. To test for the presence of EBV, in situ hybridization (ISH) was performed on paraffin sections with 
Table I. Clinicopathological characteristics of patients.

\begin{tabular}{lccc}
\hline Characteristics & $\begin{array}{c}\text { GCLS } \\
(\mathrm{n}=104)\end{array}$ & $\begin{array}{c}\text { non-GCLS } \\
(\mathrm{n}=29)\end{array}$ & P-value \\
\hline Age (years) & 62.0 & 58.4 & 0.1605 \\
Gender (M/F) & $78 / 26$ & $16 / 13$ & 0.0381 \\
Tumor size $\left(\mathrm{cm}^{2}\right)$ & 4.29 & 5.97 & 0.0084 \\
Location & & & \\
$\quad$ Upper/middle/low & $54 / 29 / 21$ & $7 / 15 / 7$ & 0.0194 \\
Histology W/M/P & $5 / 25 / 74$ & $0 / 3 / 26$ & 0.1083 \\
Depth of invasion & & & \\
M, SM/MP, SS & $48 / 56$ & $18 / 11$ & 0.4306 \\
Lymphatic invasion +/- & $1 / 103$ & $2 / 27$ & 0.1197 \\
Vessel invasion +/- & $22 / 82$ & $11 / 18$ & 0.0644 \\
Lymph node +/- & $10 / 94$ & $7 / 22$ & 0.0383 \\
EBER +/- & $78 / 26$ & $0 / 29$ & $<0.0001$ \\
\hline
\end{tabular}

GCLS, gastric carcinoma with lymphoid stroma; non-GCLS, gastric carcinoma without lymphoid stroma; M, male; F, female; histology: $\mathrm{W}$, well-differentiated; $\mathrm{M}$, moderately-differentiated; $\mathrm{P}$, poorlydifferentiated; depth of invation: M, mucosa; SM, submucosa; MP, muscularis propria; SS, subserosa.

an EBV-encoded RNA (EBER)-specific peptide nucleic acid (PNA) probe and a PNA ISH detection kit (DakoCytomation, Carpinteria, CA, USA), according to the manufacturer's instructions, as previously described (15). EBV-positive nasopharyngeal carcinoma (non-keratinizing undifferentiated carcinoma) was used as the positive control.

Immunohistochemical staining and evaluation. Tumor samples were fixed with $10 \%$ formaldehyde, embedded in paraffin, and sectioned into 4- $\mu \mathrm{m}$ slices. Immunohistochemical staining was performed using the streptavidin-biotin-peroxidase method (Histofine ${ }^{\circledR}$ staining kit; Nichirei Co., Tokyo, Japan). The primary antibodies used in this study were rabbit polyclonal anti-ezrin (dilution, 1:200; Cell Signaling Technology, Inc., Beverly, MA, USA), and rabbit polyclonal anti-phospho-ezrin (dilution, 1:200; Cell Signaling Technology, Inc.). The phosphorylated form identified the endogenous levels of ezrin, radixin and moesin only when phosphorylated at Thr567 of ezrin, Thr564 of radixin or Thr558 of moesin, respectively. Following the inhibition of endogenous peroxidase in a $3 \%$ $\mathrm{H}_{2} \mathrm{O}_{2}$-methanol solution for $15 \mathrm{~min}$ and antigen retrieval microwave irradiation in citrate buffer $(\mathrm{pH}$ 6.0) for the two antibodies, the sections were exposed to each primary antibody at $37^{\circ} \mathrm{C}$ overnight. The sections were then reacted in 3,3'-diaminobenzidine, counterstained with hematoxylin and mounted. Scoring of the immunohistochemical results was performed by two pathologists (TT and SA), who were unaware of the clinical data. Immunohistochemical staining was evaluated in the carcinoma cell component. The proportion of positive cells was counted in $>1,000$ carcinoma cells and was recorded as a percentage.
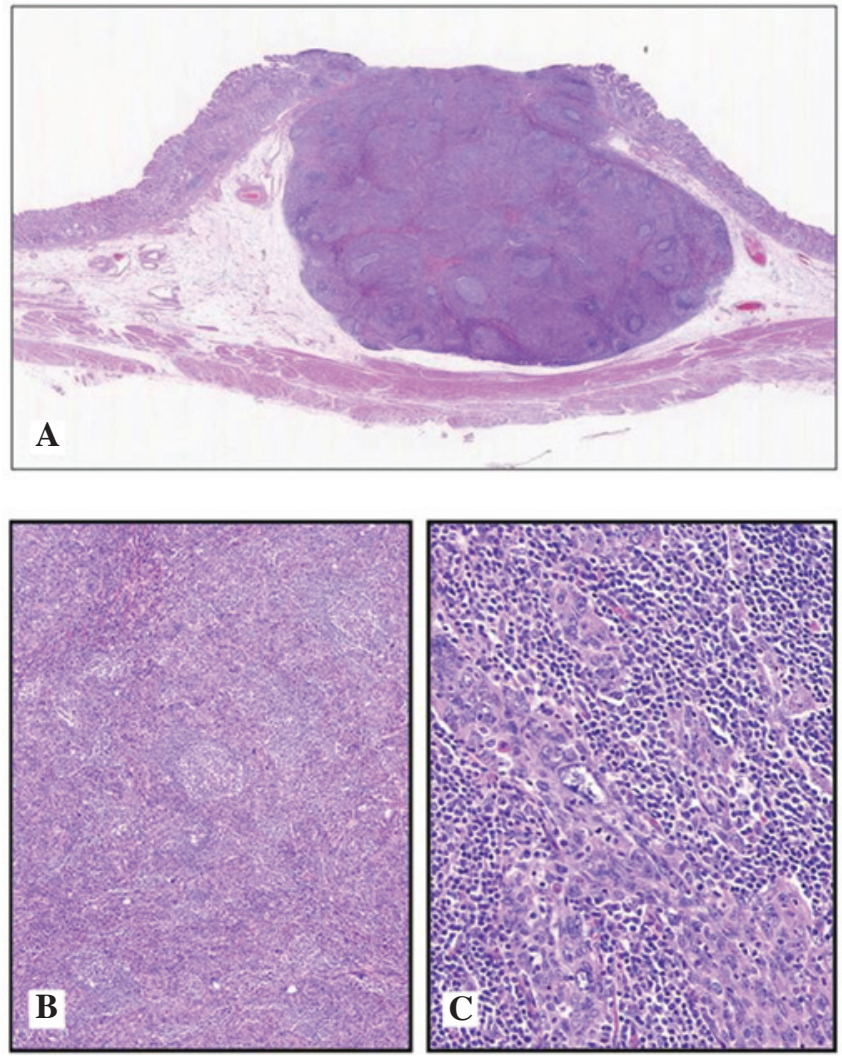

Figure 1. (A) Representative gastric carcinoma shows a well-circumscribed and expansive growth pattern. (B) Dense and diffuse lymphoid infiltration together with lymphoid follicles. (C) Lace-like or small-nested proliferative pattern of carcinoma cells with irregular nuclei.



Figure 2. Expression of (A and B) EBER, (C and D) ezrin and (E and F) p-ERM. The expression is observed in (A, C and E) GCLS, but not in (B, D and F) non-GCLS. EBER, EBV-encoded RNA; p-ERM, phosphorylatedezrin/radixin/moesin. 
Table II. Correlation between ezrin or p-ERM expression and clinicopathological characteristics.

\begin{tabular}{|c|c|c|c|c|c|c|}
\hline \multirow[b]{2}{*}{ Characteristics } & \multicolumn{3}{|c|}{ Ezrin } & \multicolumn{3}{|c|}{ p-ERM } \\
\hline & $\begin{array}{l}>30 \% \\
(\mathrm{n}=95)\end{array}$ & $\begin{array}{l}<30 \% \\
(\mathrm{n}=9)\end{array}$ & P-value & $\begin{array}{l}>30 \% \\
(\mathrm{n}=45)\end{array}$ & $\begin{array}{l}<30 \% \\
(\mathrm{n}=59)\end{array}$ & P-value \\
\hline Age (years) & 62.1 & 60.7 & 0.7238 & 60.7 & 62.9 & 0.3407 \\
\hline Gender (M/F) & $70 / 25$ & $8 / 1$ & 0.4444 & $31 / 14$ & $47 / 12$ & 0.2088 \\
\hline Tumor size $\left(\mathrm{cm}^{2}\right)$ & 4.446 & 2.656 & 0.0867 & 4.800 & 3.903 & 0.1313 \\
\hline \multicolumn{7}{|l|}{ Location } \\
\hline Upper/middle/low & $47 / 29 / 19$ & $6 / 0 / 3$ & 0.1412 & $25 / 15 / 5$ & $28 / 14 / 17$ & 0.0840 \\
\hline Histology W/M/P & $4 / 23 / 68$ & $1 / 2 / 6$ & 0.6519 & $4 / 10 / 31$ & $1 / 15 / 43$ & 0.2329 \\
\hline \multicolumn{7}{|l|}{ Depth of invasion } \\
\hline M, SM/MP, SS & $42 / 53$ & $6 / 3$ & 0.2962 & $18 / 27$ & $30 / 29$ & 0.2716 \\
\hline Lymphatic invasion +/- & $20 / 75$ & $2 / 7$ & 0.9346 & $9 / 36$ & $13 / 46$ & 0.8013 \\
\hline Vessel invasion +/- & $0 / 95$ & $1 / 8$ & 0.0865 & $1 / 44$ & $0 / 59$ & 0.4327 \\
\hline Lymph node +/- & $10 / 85$ & $0 / 9$ & 0.3059 & $8 / 37$ & $2 / 57$ & 0.0187 \\
\hline
\end{tabular}

p-ERM, phosphorylated ezrin/radixin/moesin; M, male; F, female; histology: W, well-differentiated; M, moderately differentiated; P, poorlydifferentiated; depth of invasion: $\mathrm{M}$, mucosa; SM, submucosa; MP, muscularis propria; SS, subserosa.
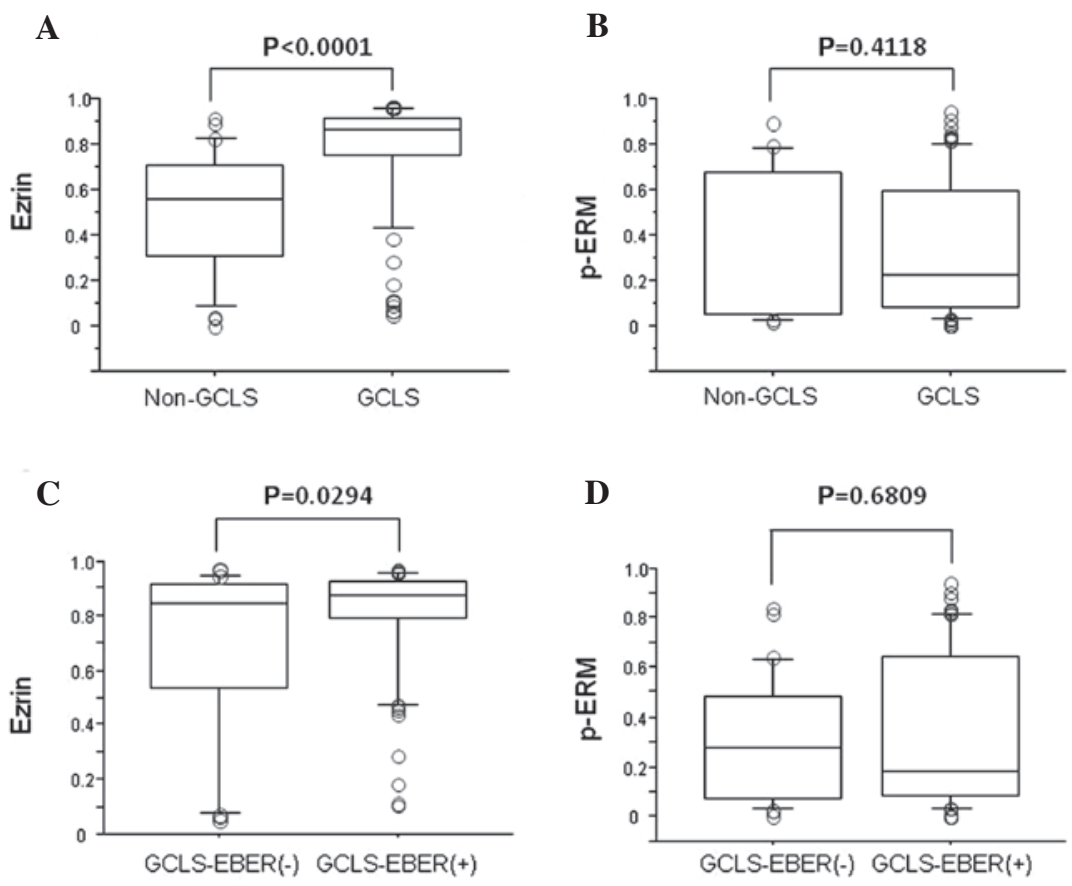

Figure 3. Comparison of (A) ezrin and (B) p-ezrin expression between non-GCLS and GCLS. Comparison of (C) ezrin and (D) p-ezrin expression between EBER-negative and EBER-positive GCLS. GCLS, gastric carcinoma with lymphoid stroma; non-GCLS, gastric carcinoma without lymphoid stroma; p-ERM, phosphorylated-ezrin/radixin/moesin; EBER, EBV-encoded RNA.

Statistical analysis. For the statistical analysis, the expression of ezrin or p-ezrin in carcinoma cells was identified as a high-expression group, when the cytoplasm or membrane staining showed $>30 \%$ of carcinoma cells. Statistical analysis of group differences was carried out using the Chi-square, Fisher's exact and Student's-t tests. $\mathrm{P}<0.05$ was considered statistically significant.

\section{Results}

Clinicopathological characteristics of GCLS and non-GCLS patients. The clinicopathological characteristics of patients are summarized in Table I. The GCLS group included 74 males and 26 females with a median age of 62.0 years (range, 37-90), whereas the non-GCLS group included 16 males and 13 females 
with a median age of 58.4 years (range, 39-82) $(\mathrm{P}=0.0381)$. The tumor size of GCLS was larger compared to that of non-GCLS ( $\mathrm{P}=0.0084)$. There were 54, 29 and 21 GCLS cases from the upper, middle and lower stomach, as well as 7,15 and 7 non-GCLS cases from the upper, middle and lower stomach, respectively $(\mathrm{P}=0.0194)$. Lymph node metastasis in GCLS was markedly infrequent compared to non-GCLS $(\mathrm{P}=0.0383)$.

GCLS cases were divided into EBER-positive $(n=76)$ (Fig. 2A) and -negative cases $(n=28)$ by in situ hybridization. By contrast, non-GCLS cases included no EBER-positive cases (Fig. 2B) $(\mathrm{P}<0.0001)$.

Ezrin and p-ezrin expression in GCLS and non-GCLS. Positive ezrin expression was diffusely observed in the cytoplasm and membrane of carcinoma cells (Fig. 2C), whereas p-ezrin was predominantly detected in the membrane of carcinoma cells alone (Fig. 2E). Ezrin expression was markedly higher in GCLS compared to non-GCLS $(\mathrm{P}<0.0001)$ (Fig. 3A), although there were no differences in the p-ezrin expression in GCLS and non-GCLS (Fig. 3B). Ezrin expression was higher in EBV-positive GCLS compared to -negative GCLS $(\mathrm{P}=0.0294)$ (Fig. 3C), although there was no difference in p-ezrin expression in EBV-positive and -negative GCLS (Fig. 3D).

Correlation between ezrin/p-ezrin expression and clinicopathological characteristics of GCLS patients. The correlation between the expression of ezrin or p-ezrin and the clinicopathological patient characteristics is summarized in Table II. High expression of ezrin and p-ezrin ( $>30 \%$ of positive carcinoma cells) of GCLS was observed in 95/104 $(91 \%)$ and in 45/104 (43\%) of cases, respectively. Forty five of p-ezrin-positive cases included 42 ezrin-positive and 3 -negative cases. No significant differences were observed between ezrin expression and the clinicopathological characteristics, however, the high expression of ezrin tended to correspond to a larger tumor size $(\mathrm{P}=0.0867)$. Only p-ezrin in GCLS was associated with positive lymph node metastasis $(\mathrm{P}=0.0187)$.

\section{Discussion}

EBV-associated gastric carcinoma accounts for $\sim 10 \%$ of gastric carcinomas and histologically resembles GCLS (16). Consistent with a previous study, the GCLS cases of this study demonstrated a male predominance, a greater tendency towards being located in the fundic or upper gastric regions and a lower frequency of lymph node metastasis, compared to the control cases (17). Moreover, 75\% of GCLS cases demonstrated EBER positivity. Taken together, these findings indicate that the cases examined in this study might be characterised as typical GCLS cases.

Molecular abnormalities of EBV-associated gastric carcinomas remain unknown, whereas the $\mathrm{CpG}$ island methylation in the promoter region of PTEN gene has been previously documented (18). Endo et al (14) have demonstrated EBV LMP1 to be associated with ezrin phosphorylation in nasopharyngeal carcinoma, whereas the present is the first study to indicate that ezrin expression is correlated with GCLS and EBER positivity.

Ezrin is widely expressed in malignant tumors, including gastric $(12,13)$ and colon cancers (19), hepatocellular carcinoma (20), ovarian (21) as well as breast cancer (22), and is associated with poor prognosis. Furthermore, ezrin expression is correlated with an early recurrence of hepatocellular carcinoma (22), invasion of pancreatic adenocarcinoma (23) and lymph node metastasis of prostate cancer (24) and nasopharyngeal carcinoma (25).

According to a comparison between ezrin or p-ezrin exression and clinicopathological characteristics, only lymph node metastasis is associated with p-ezrin. In the present study, anti-ezrin unphosphorylated type and anti-ERM [ezrin (Thr567)/radixin (Thr564)/moesin (Thr558), Cell Signaling Technology, Inc.] antibody was used as the anti-ezrin phosphorylated form at the Thr567 site, also used as the p-ezrin antibody at Thr567 in previously published studies $(26,27)$. The phosphorylation of residual Thr567 in ezrin alters the protein to expose its binding sites (28) thus resulting in oncogene-induced transformation (29). The phosphorylation at Thr567 may not be necessary for osteosarcoma metastasis (27). In addition, phosphorylation at Tyr353, but not at Thr567, is associated with lymph node metastasis of pancreatic adenocarcinoma (30). However, Tang et al (27) have reported that ezrin phosphorylation at Thr567 may induce lymph node metastasis of nasopharyngeal carcinomas. The findings in the present study were consistent with the hypothesis that p-ezrin at Thr567 is crucial in lymph node metastasis. Notably, nasopharyngeal carcinoma and GCLS are commonly associated with EBV infection.

Based on recent studies of targeting for ezrin, reduction of the ezrin gene by RNA interference (RNAi) may inhibit the migration and invasion of human gastric cancer cells (31). Berberine also inhibits metastasis of nasopharyngeal carcinoma cells by targeting Rho kinase-mediated ezrin phosphorylation at Thr567 (27). These findings suggest that the molecular target therapy of ezrin and its phosphorylation should be considered to be EBV-associated GCLS.

In conclusion, ezrin expression is correlated with GCLS with EBV infection, while the phosphorylation of ezrin is essential in the lymph node metastasis of GCLS.

\section{References}

1. Watanabe H, Enjoji $\mathbf{M}$ and Imai T: Gastric carcinoma with lymphoid stroma. Its morphologic characteristics and prognostic correlations. Cancer 38: 232-243, 1976.

2. Weiss LM, Movahed LA, Butler AE, et al: Analysis of lymphoepithelioma and lymphoepithelioma-like carcinomas for Epstein-Barr viral genomes by in situ hybridization. Am J Surg Pathol 13: 625-631, 1989.

3. Nakamura S, Ueki T, Yao T, et al: Epstein-Barr virus in gastric carcinoma with lymphoid stroma. Special reference to its detection by the polymerase chain reaction and in situ hybridization in 99 tumors, including a morphologic analysis. Cancer 73: 2239-2249, 1994.

4. Matsunou H, Konishi F, Hori H, et al: Characteristics of Epstein-Barr virus-associated gastric carcinoma with lymphoid stroma in Japan. Cancer 77: 1998-2004, 1996.

5. Oda K, Tamaru J, Takenouchi T, et al: Association of Epstein-Barr virus with gastric carcinoma with lymphoid stroma. Am J Pathol 143: 1063-1071, 1993.

6. Burke AP, Yen TS, Shekitka KM, et al: Lymphoepithelial carcinoma of the stomach with Epstein-Barr virus demonstrated by polymerase chain reaction. Mod Pathol 3: 377-380, 1990.

7. Vaheri A, Carpén O, Heiska L, et al: The ezrin protein family: membrane-cytoskeleton interactions and disease associations. Curr Opin Cell Biol 9: 659-666, 1997.

8. Akisawa N, Nishimori I, Iwamura T, et al: High levels of ezrin expressed by human pancreatic adenocarcinoma cell lines with high metastatic potential. Biochem Biophys Res Commun 258: 395-400, 1999. 
9. Sarrió D, Rodríguez-Pinilla SM, Dotor A, et al: Abnormal ezrin localization is associated with clinicopathological features in invasive breast carcinomas. Breast Cancer Res Treat 98: 71-79, 2006.

10. Khanna C, Wan X and Bose S: The membrane-cytoskeleton linker ezrin is necessary for osteosarcoma metastasis. Nat Med 10: 182-186, 2004

11. Bal N, Yildirim S, Nursal TZ, et al: Association of ezrin expression in intestinal and diffuse gastric carcinoma with clinicopathological parameters and tumor type. World J Gastroenterol 13: 3726-3729, 2007.

12. Zhao J, Zhang X and Xin Y: Up-regulated expression of Ezrin and c-Met proteins are related to the metastasis and prognosis of gastric carcinomas. Histol Histopathol 26: 1111-1120, 2011.

13. Li L, Wang YY, Zhao ZS and Ma J: Ezrin is associated with gastric cancer progression and prognosis. Pathol Oncol Res 17: 909-915, 2011.

14. Endo K, Kondo S, Shackleford J, et al: Phosphorylated ezrin is associated with EBV latent membrane protein 1 in nasopharyngeal carcinoma and induces cell migration. Oncogene 28: $1725-1735,2009$.

15. Yamamoto H, Kohashi K, Oda Y, et al: Absence of human herpesvirus- 8 and Epstein-Barr virus in inflammatory myofibroblastic tumor with anaplastic large cell lymphoma kinase fusion gene. Pathol Int 56: 584-590, 2006.

16. Fukayama M: Epstein-Barr virus and gastric carcinoma. Pathol Int 60: 337-350, 2010

17. van Beek J,zur Hausen A, Klein Kranenbarg E, et al: EBV-positive gastric adenocarcinomas: a distinct clinicopathologic entity with a low frequency of lymph node involvement. J Clin Oncol 22: 664-670, 2004.

18. Hino R, Uozaki H, Murakami N, et al: Activation of DNA methyltransferase 1 by EBV latent membrane protein $2 \mathrm{~A}$ leads to promoter hypermethylation of PTEN gene in gastric carcinoma. Cancer Res 69: 2766-2774, 2009.

19. Elzagheid A, Korkeila E and Bendardaf R: Intense cytoplasmic ezrin immunoreactivity predicts poor survival in colorectal cancer. Hum Pathol 39: 1737-1743, 2008.
20. Kang YK, Hong SW and Lee H, et al: Prognostic implications of ezrin expression in human hepatocellular carcinoma. Mol Carcinog 49: 798-804, 2010.

21. Moilanen J, Lassus H, Leminen A, et al: Ezrin immunoreactivity in relation to survival in serous ovarian carcinoma patients. Gynecol Oncol 90: 273-281, 2003.

22. Okamura D, Ohtsuka M, Kimura F, et al: Ezrin expression is associated with hepatocellular carcinoma possibly derived from progenitor cells and early recurrence after surgical resection. Mod Pathol 21: 847-855, 2008.

23. Kocher HM, Sandle J, Mirza TA, et al: Ezrin interacts with cortactin to form podosomal rosettes in pancreatic cancer cells. Gut 58: 271-284, 2009

24. Pang J, Liu WP, Liu XP, et al: Profiling protein markers associated with lymph node metastasis in prostate cancer by DIGE-based proteomics analysis. J Proteome Res 9: 216-226, 2010.

25. Wang L, Lin GN, Jiang XL, et al: Expression of ezrin correlates with poor prognosis of nasopharyngeal carcinoma. Tumour Biol 32: 707-712, 2011.

26. Di Cristofano C, Leopizzi M, Miraglia A, et al: Phosphorylated ezrin is located in the nucleus of the osteosarcoma cell. Mod Pathol 23: 1012-1020, 2010.

27. Tang F, Wang D, Duan C, et al: Berberine inhibits metastasis of nasopharyngeal carcinoma $5-8 \mathrm{~F}$ cells by targeting Rho kinase-mediated Ezrin phosphorylation at threonine 567. J Biol Chem 284: 27456-27466, 2009.

28. Zhu L, Zhou R, Mettler S, et al: High turnover of ezrin T567 phosphorylation: conformation, activity, and cellular function. Am J Physiol Cell Physiol 293: C874-C884, 2007.

29. Tran Quang C, Gautreau A, Arpin M, et al: Ezrin function is required for ROCK-mediated fibroblast transformation by the Net and Dbl oncogenes. EMBO J 19: 4565-4576, 2000.

30. Cui Y, Li T, Zhang D and Han J: Expression of Ezrin and phosphorylated Ezrin (pEzrin) in pancreatic ductal adenocarcinoma. Cancer Invest 28: 242-247, 2010.

31. Wang HJ, Zhu JS, Zhang Q, et al: RNAi-mediated silencing of ezrin gene reverses malignant behavior of human gastric cancer cell line SGC-7901. J Dig Dis 10: 258-264, 2009. 